\title{
Routine preoperative laboratory testing in elective pediatric cardiothoracic surgery is largely unnecessary
}

\author{
R. Michael Nieto, MD, Luis E. De León, MD, Daniel Trujillo Diaz, MD, Kimberly A. Krauklis, CRNP, and \\ Charles D. Fraser, Jr, MD
}

\begin{abstract}
Objective: Routine preoperative laboratory testing (RLT) is common practice in pediatric cardiothoracic surgery and is associated with significant cost burden to patients and families. We sought to examine the value of RLT in patients undergoing elective pediatric cardiothoracic surgery.
\end{abstract}

Methods: We conducted a retrospective study of all scheduled elective pediatric cardiothoracic surgery at our institution from 2012 to 2014. Inpatients were excluded. Patient charts were reviewed to obtain preoperative laboratory values and determine relationship to case cancellation. RLT includes complete blood count, prothrombin time, partial thromboplastin time, urinalysis, 7 chemistry metabolic panel, electrocardiogram, and 2-view chest radiograph.

Results: RLT was completed for 1106 scheduled elective cases. Six $(0.5 \%)$ cancellations were related to abnormal preoperative laboratory test results: $5 \mathrm{com}-$ plete blood counts and 1 urinalysis. Hospital charge for RLT averaged \$2064 per patient. Based on this incidence, we estimated that 184 routine preoperative laboratory tests, which generated a total hospital charge of $\$ 379,776$, were required to capture 1 abnormal test significant enough to cancel surgery. An estimated charge of $\$ 2,169,552$ was generated on prothrombin time, partial thromboplastin time, 7 chemistry metabolic panel, electrocardiogram, and 2-view chest radiograph, and none of these tests resulted in a cancellation.

Conclusions: RLT does not significantly impact decision-making in elective pediatric cardiothoracic surgery. The decision to order a specific screening test should be clinically driven. Selective preoperative laboratory testing may have a positive impact on healthcare costs without affecting outcomes. (J Thorac Cardiovasc Surg 2017;153:678-85)

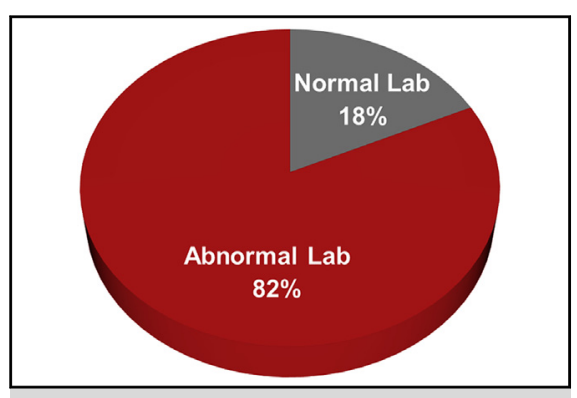

Laboratory values of pediatric patients undergoing elective cardiothoracic surgery $(n=993)$. Of the 993 pediatric patients who underwent elective cardiothoracic surgery at our institution, $819(82 \%)$ had at least 1 routine preoperative laboratory value outside of our institution's normal reference range.

\section{Central Message}

Selective preoperative laboratory testing may have a positive impact on healthcare costs without affecting outcomes.

\section{Perspective \\ Routine preoperative laboratory testing does not appear to significantly impact decision- making in elective pediatric cardiothoracic sur- gery. The majority of pediatric patients undergo elective cardiothoracic surgery with at least one laboratory value outside of normal reference ranges. Selective preoperative laboratory testing may have a positive impact on health- care costs without affecting outcomes. Selec- tive preoperative laboratory testing may have a positive impact on healthcare costs without affecting outcomes.}

See Editorial Commentary page 686.

See Editorial page 677.
From the Division of Congenital Heart Surgery and Department of Surgery, Texas Children's Hospital, Houston, Tex.

Read at the 96th Annual Meeting of The American Association for Thoracic Surgery, May 14-18, 2016, Baltimore, Maryland.

Received for publication May 25, 2016; revisions received Sept 16, 2016; accepted for publication Oct 5, 2016; available ahead of print Jan 7, 2017.
Address for reprints: Charles D. Fraser, Jr, MD, Congenital Heart Surgery, Texas Children's Hospital, 6621 Fannin St, MC19345H, Houston, TX 77030 (E-mail: CDFraser@texaschildrens.org). $0022-5223 / \$ 36.00$

Copyright $\odot 2016$ Published by Elsevier Inc. on behalf of The American Association for Thoracic Surgery http://dx.doi.org/10.1016/j.jtcvs.2016.10.082 

Abbreviations and Acronyms
$\mathrm{CBC}=$ complete blood count
Chem7 $=7$ chemistry metabolic panel
CXR = chest radiograph
$\mathrm{ECG}=$ electrocardiogram
$\mathrm{PT}=$ prothrombin time
PTT $=$ partial thromboplastin time
RLT = routine preoperative laboratory testing
$\mathrm{UA}=$ urinalysis
UTI = urinary tract infection

\section{Scanning this QR code will take you to the article title page.}

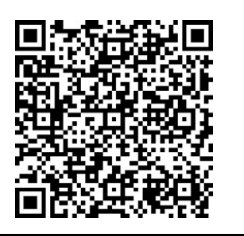

Performing routine preoperative laboratory testing (RLT) is standard in pediatric cardiothoracic surgery as part of preanesthetic evaluation to determine fitness for anesthesia and identify patients at increased risk of perioperative complications. ${ }^{1,2}$ The probability of finding an abnormality significant enough to warrant cancellation of surgery through preoperative testing is small, however, and results in significant cost burden to patients and families. ${ }^{3}$ The use of RLT in pediatric patients undergoing elective, lowrisk, noncardiac surgery has been the focus of several clinical researchers and specialty societies during the past 3 decades. The resultant societal guidelines and literature have shown that in the absence of a clinical indication, routine preoperative tests in patients undergoing elective, lowrisk, noncardiac surgery are expensive and not useful. ${ }^{2-6}$ Despite the guidelines and solid evidence indicating that RLT is unnecessary, routine preoperative tests continue to be ordered frequently, with much variation as to which tests are ordered across institutions, specialties, and individual providers. ${ }^{6-10}$

Although several clinical investigators have evaluated the utility of routine preoperative testing in patients undergoing low-risk, elective, and/or ambulatory surgery, few have examined the impact routine preoperative testing has on greater-risk elective surgery. There is limited evidence in the literature regarding the clinical benefit and costeffectiveness of routine screening tests before pediatric cardiothoracic surgery in the absence of specific clinical or historical (eg, history of bleeding diathesis) indication, and whether the series of screening tests affect the management plan of elective surgery. We sought to examine the value of routine preoperative laboratory testing in children undergoing elective cardiothoracic surgery and the effect routine preoperative laboratory testing has on the decision-making process of surgical timing.

\section{METHODS}

\section{Study Population}

We conducted a retrospective review of all elective, investigated pediatric cardiothoracic surgery cases at Texas Children's Hospital from January 2012 through December 2014. For this study, we defined an investigated case as a scheduled patient, either cancelled or completed, who underwent RLT.

To identify all investigated cases, 2 separate search queries were performed. First, patient electronic medical records were queried for cancellations of scheduled cases. A case was considered cancelled if it had been scheduled previously but not performed on the planned date. Patient charts were reviewed to obtain the reason for cancellation, patient's age, sex, date of admission, date of procedure, and type of procedure. Case cancellations involving inpatients were excluded. Elective cases that were cancelled and underwent RLT $(\mathrm{n}=234)$ were selected for further review. An additional query was then carried out to identify all completed, elective pediatric cardiothoracic surgery cases that underwent RLT $(n=993)$. Cases that were completed more than 1 day after admission were excluded. In total, 1106 unique pediatric cardiothoracic surgery cases were investigated, with some overlap between elective cases that were performed on the scheduled date and cancelled cases that were performed days later that did not undergo repeat preoperative laboratory testing $(\mathrm{n}=121)$ (Figure 1).

\section{Laboratory Testing}

Preoperative laboratory data were obtained on all patients who were cancelled or underwent surgery from January 2012 through December 2014 and met our inclusion criteria. At our institution, all patients undergo RLT including a complete blood count (CBC), prothrombin time (PT), partial thromboplastin time (PTT), urinalysis (UA), 7 chemistry metabolic panel (Chem7), electrocardiogram (ECG), and 2-view chest radiograph (CXR).

The breakdown of RLT values used for analysis are as follows. Values obtained from the CBC included white blood cell count, hemoglobin, hematocrit, platelet count, and absolute neutrophil count. Values obtained from the Chem7 included sodium, potassium, chloride, glucose, blood urea nitrogen, and creatinine. Values obtained from coagulation tests included activated PTT, PT, and international normalized ratio. Results obtained from the UA included white blood cell count, leukocyte esterase, nitrites, glucose, ketones, blood, and bacteria.

Preoperative laboratory tests were reviewed to identify any abnormality and determine whether the abnormality led to cancellation of the scheduled surgical procedure. An abnormal result was defined as a value that did not fall within the standardized reference range as established by our hospital laboratory. UA was considered abnormal if (1) there was suspicion of a urinary tract infection (UTI), defined by the presence of leukocyte esterase and nitrites or $>4$ leukocytes per high-power field and bacteriuria, ${ }^{11}$ or (2) urine contained ketones, protein, glucose, or blood. ${ }^{12}$

\section{Analysis}

The primary outcome of this study was the percentage of surgical cases cancelled specifically because of abnormal routine preoperative laboratory results. We also evaluated the percentage of surgical cases that were not cancelled despite having preoperative laboratory values outside of normal reference ranges. Interquartile ranges were calculated for the differences between the high or low abnormal laboratory values and the upper or lower limits of their respective reference ranges. For this study, we defined outliers as values above the 95 th percentile or below the 5 th percentile.

An average of charges for each test was obtained from the hospital's financial administration. Hospital charges ranged between $\$ 53.00$ and $\$ 563.00$ per test. Because this was a retrospective study and because 


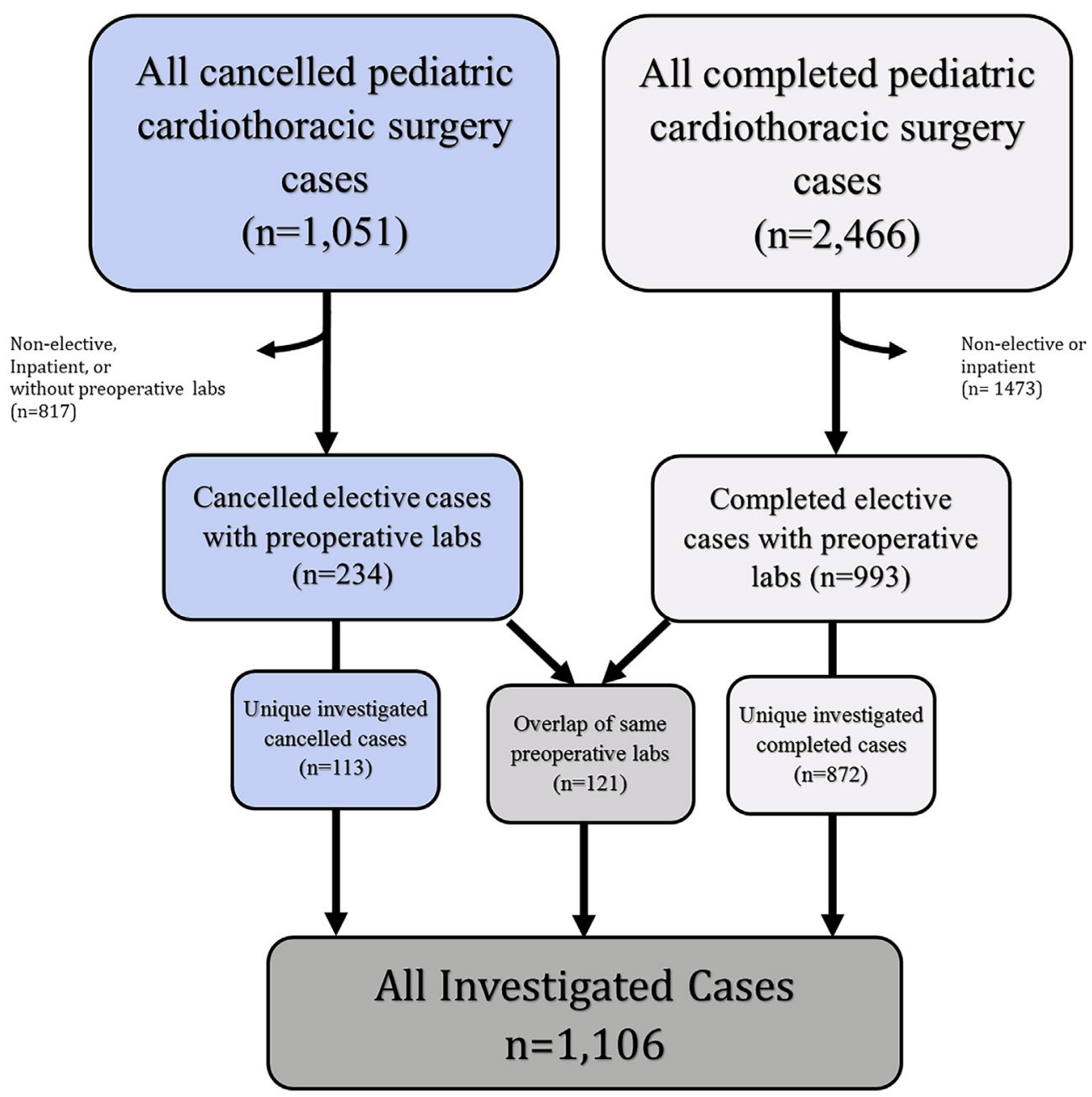

FIGURE 1. Patients undergoing preoperative laboratory testing.

charges varied over time, we were not able to verify the exact charge of each individual test completed on the patients in our cohort, and therefore are presented as averages.

Data are described as percentages and medians with ranges. This study was approved by the institutional review board of Baylor College of Medicine, and informed consent was waived.

\section{RESULTS}

Between 2012 and 2014, a total of 3517 pediatric cardiothoracic surgery cases were scheduled at Texas Children's Hospital, of which 2466 were performed and 1051 were cancelled. Our final cohort comprised 1106 scheduled elective patients who underwent RLT. Of these, 993 cases were performed and 234 cases were cancelled. A total of 121 cases were included in both groups because they underwent RLT before cancellation and underwent their deferred surgical procedure a few days later without repeating the laboratory testing (Figure 1).

Among the 993 completed cases, $2(0.2 \%)$ patients did not have Chem7 results, $22(2.2 \%)$ did not have

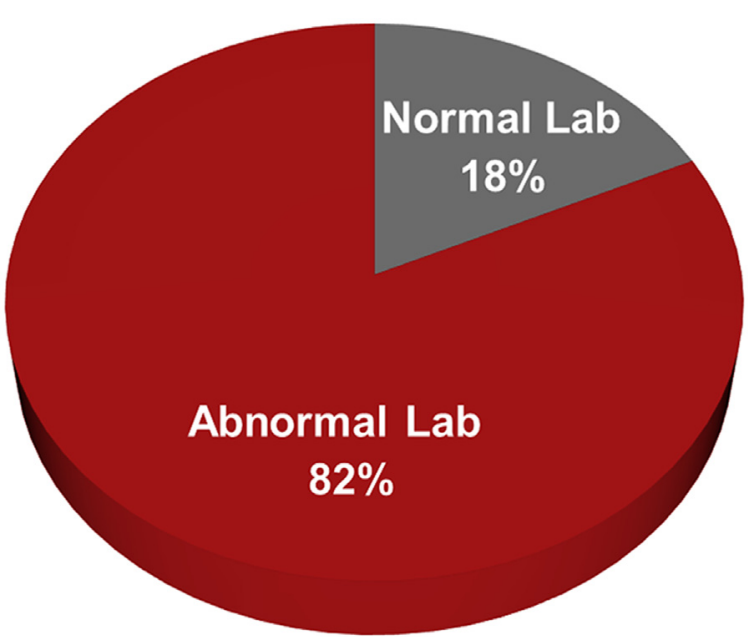

FIGURE 2. Laboratory values of pediatric patients undergoing elective cardiothoracic surgery $(\mathrm{n}=993)$. Of the 993 pediatric patients who underwent elective cardiothoracic surgery at our institution, $819(82 \%)$ had at least 1 routine preoperative laboratory value outside of our institution's normal reference range. 


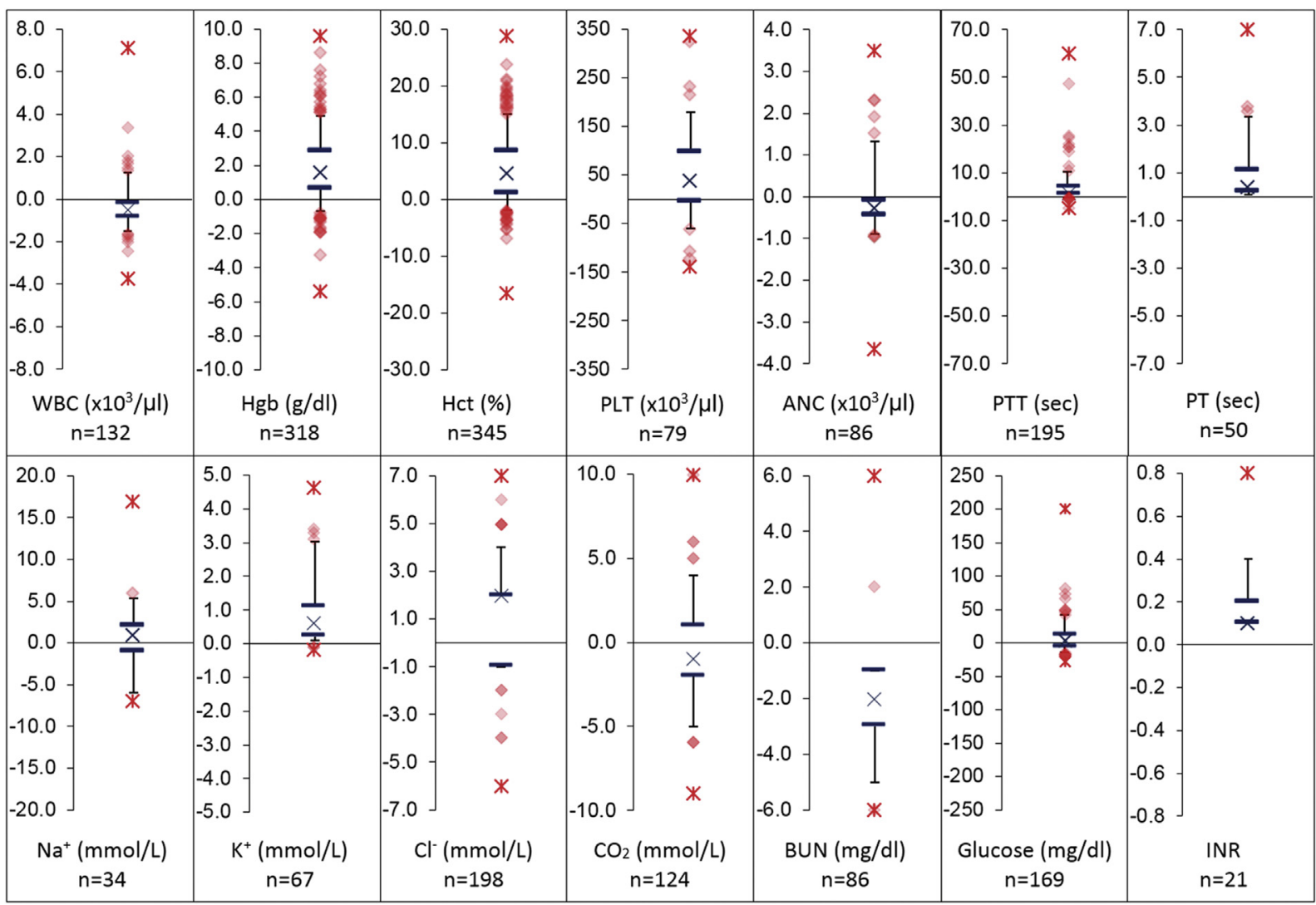

FIGURE 3. Differences between abnormal laboratory values and their reference ranges. The differences between a patient's abnormal laboratory result and the lower or upper limit of their corresponding reference range values are plotted. Negative values represent abnormal results below the reference range, and positive values represent results above the reference range. The median of the differences is represented with an " $\mathrm{X}$ " and horizontal markers are used for $\mathrm{Q} 1$ and Q3. The whiskers represent the 5th and 95th percentiles. Upper and lower outliers are represented outside of the whiskers. WBC, White blood cell count; $\mathrm{Hg}$, hemoglobin; Hct, hematocrit; PLT, platelets; ANC, absolute neutrophil count; PTT, partial thromboplastin time; PT, prothrombin time; BUN, blood urea nitrogen; INR, international normalized ratio.

UA results, and $34(3.4 \%)$ did not have coagulation test results. The main reasons for not having these results preoperatively were attributed to an insufficient or clotted blood sample or the sample was unable to be obtained after several attempts. The majority of these missing coagulation tests and UAs were completed in the operating room either before or near the start of the procedure. These laboratory test results were not

TABLE 1. Distribution of laboratory values outside of the reference range

\begin{tabular}{|c|c|c|c|}
\hline & Upper outliers $>95$ th percentile & Lower outliers $<$ 5th percentile & Median (range) \\
\hline Sodium, mmol/L & 2 & 2 & $1(-7$ to 17$)$ \\
\hline Potassium, mmol/L & 4 & 3 & $0.6(-0.2$ to 4.6$)$ \\
\hline Chloride, $\mathrm{mmol} / \mathrm{L}$ & 6 & 6 & $2(-6$ to 7$)$ \\
\hline $\mathrm{CO}_{2}, \mathrm{mmol} / \mathrm{L}$ & 6 & 4 & $-1(-9$ to 10$)$ \\
\hline BUN, mg/dL & 2 & 2 & $-2(-6$ to 6$)$ \\
\hline Glucose, $\mathrm{mg} / \mathrm{dL}$ & 9 & 9 & $3(-28$ to 201$)$ \\
\hline $\mathrm{WBC}, \times 10^{3} / \mu \mathrm{L}$ & 7 & 7 & $-0.49(-3.71$ to 7.14$)$ \\
\hline Hemoglobin, g/dL & 16 & 15 & $1.6(-5.4$ to 9.6$)$ \\
\hline Hematocrit, \% & 18 & 17 & $4.6(-16.6$ to 28.7$)$ \\
\hline Platelets, $\times 10^{3} / \mu \mathrm{L}$ & 4 & 4 & $38(-138$ to 337$)$ \\
\hline $\mathrm{ANC}, \times 10^{3} / \mu \mathrm{L}$ & 5 & 5 & $-0.27(-3.6$ to 3.4$)$ \\
\hline PTT, s & 10 & 10 & $1.7(-4.9$ to 60.1$)$ \\
\hline PT, s & 3 & $0^{*}$ & $0.4(0.1-7)$ \\
\hline INR & 1 & $0 *$ & $0.1(0.1-0.8)$ \\
\hline
\end{tabular}

$B U N$, Blood urea nitrogen; $W B C$, white blood cell count; $A N C$, absolute neutrophil count; $P T T$, partial thromboplastin time; $P T$, prothrombin time; INR, international normalized ratio. *No abnormally low values were registered. 
TABLE 2. Characteristics of case cancellations specifically caused by abnormal routine preoperative laboratory results

\begin{tabular}{lllll}
\hline Patient & $\begin{array}{c}\text { Laboratory } \\
\text { test }\end{array}$ & $\begin{array}{c}\text { Abnormal laboratory values } \\
(\text { WBC: } \times \mathbf{1 0} / \boldsymbol{\mathbf { m }} \mathbf{L}, \mathbf{A N C}: \times \mathbf{1 0} \boldsymbol{\mathbf { 3 }} / \boldsymbol{\mu} \mathbf{L})\end{array}$ & $\begin{array}{c}\text { Scheduled } \\
\text { procedure }\end{array}$ \\
\hline 1 & CBC & WBC: 2.89, ANC: 0.84 & PA-IVS & Fontan \\
2 & CBC & WBC: 2.39, ANC: 0.83 & DORV, VSD & Fontan \\
3 & CBC & WBC: 15.3, ANC: 12.64 & DORV, TGA & Fontan \\
4 & CBC & WBC: 3.0, ANC: 0.56 & Unbalanced AVSD & AVSD repair \\
5 & CBC & WBC: 16.4, ANC: 12.58 & ASD & ASD closure \\
6 & UA & UTI & VSD & VSD repair \\
\hline
\end{tabular}

WBC, White blood cell count; $A N C$, absolute neutrophil count; $C B C$, complete blood count; $P A-I V S$, pulmonary atresia with intact ventricular septum; $D O R V$, double outlet right ventricle; VSD, ventricular septal defect; $T G A$, transposition of great arteries; $A V S D$, atrioventricular septal defect; $A S D$, atrial septal defect; UA, urinalysis; UTI, urinary tract infection.

included for analysis. In total, $819(82.5 \%)$ patients had at least 1 routine preoperative laboratory value outside of our institution's normal reference range (Figure 2).

Figure 3 shows the distribution of the differences between the abnormal laboratory values and the upper and lower limits of their corresponding reference ranges. Table 1 shows the actual number of outliers, which represents those differences above the 95th percentile and those below the 5 th percentile. The median and ranges of the differences for each of the tests performed are also shown. Despite these differences, all of these patients underwent cardiothoracic surgery. Two hundred forty-nine (25\%) patients had an abnormal UA result, of which 54 were suspected UTIs and 219 had glucose, ketones, blood, and/or protein present in their urine.

Six $(0.5 \%)$ investigated elective cases were cancelled specifically because of an abnormal routine preoperative laboratory result (5 abnormal CBCs and 1 abnormal UA). Characteristics of these cancelled cases caused by abnormal RLT are summarized in Table 2. Patients 1 and 4 only underwent repeated testing with normalization of their laboratory results. Patient 2 underwent a viral panel and patient 3 continued to have abnormal laboratory results necessitating consultation to our hematology colleagues. None of these 4 patients was diagnosed with a new illness, nor did they require treatment and all underwent elective cardiothoracic surgery after normalization of their laboratory values. No further record was found after cancellation of surgery for patient 5. Patient 6 was treated with antibiotics for a suspected UTI, was later found to be culture-positive and underwent a second round of antibiotics, and ultimately underwent elective cardiothoracic surgery once a normal UA was obtained. None of the 1106 investigated elective cases were cancelled because of abnormal results in PT, PTT, international normalized ratio, Chem7, ECG, or CXR. Other reasons for cancellation included emergent cases, patient illness, parent/family request, or failure to obtain dental clearance.

Estimated hospital charges for RLT averaged \$2064. Total estimated hospital charges for RLTs completed during this study period were $\$ 2,282,784$. Based on this incidence, we estimated that 184 RLTs, which generated an estimated total hospital charge of $\$ 379,776$, were required to reveal 1 abnormal test believed significant enough to cancel surgery.

\section{DISCUSSION}

RLT of surgical patients continues to be common, although with significant variation across institutions, specialties, and physicians. The belief that all patients need RLTs is ingrained in surgical practice. These tests presumably are ordered to identify patients at increased risk of perioperative complications, to identify previously unknown illnesses, and so that physicians may alter the perioperative management strategy in an attempt to decrease morbidity and mortality. ${ }^{2}$ Despite these factors, theoretically favoring the practice of preoperative testing, the economic and psychological burden for patients and families undergoing these tests have lead us and others to question this practice. ${ }^{1-3}$

In an attempt to improve perioperative patient management and potentially reduce consultations, delays, and cancellations on the day of surgery, the American Society of Anesthesiologists Task Force on Preanesthesia Evaluation published in 2012 an update to their 2002 Practice Advisory for Preanesthesia Evaluation. Recommendations include, "Preoperative tests may be ordered, required, or performed on a selective basis for purposes of guiding or optimizing perioperative management" and should be "based on information obtained from medical records, patient interview, physical examination, and type and invasiveness of the planned procedure." ${ }^{4}$ Similarly, the American College of Radiology's most recent guidelines regarding routine chest radiography also suggested selective ordering of preoperative CXR in adults. ${ }^{13}$

Despite these recommendations, the practice of ordering RLT remains common. The factors thought to influence the continued use of potentially unnecessary preoperative testing include practice tradition, belief that other physicians want the tests done, medicolegal concerns, concern about surgical delay or cancellation, and lack of awareness of evidence and guidelines. ${ }^{8}$ Even among clinicians who are well aware of the available evidence and guidelines, applying these recommendations to their patients is not a simple task. Furthermore, attempting to apply recommendations to children becomes more challenging because the available 
evidence is predominantly adult data. The ambiguity of the available recommendations often lead the clinician to err on what is perceived to be the safe side and order extensive and potentially unnecessary routine preoperative tests. ${ }^{4}$

Our study revealed that RLT does not impact the decision-making process of surgical timing at our institution. In a population that most guidelines would recommend performing routine preoperative testing, only $6(0.5 \%)$ planned procedures in 3 years were cancelled because of abnormalities detected through RLT in otherwiseasymptomatic patients with congenital heart disease. Of all the patients who underwent their scheduled surgery, $819(82.5 \%)$ did so with at least 1 laboratory value outside of normal reference ranges. Approximately $50 \%$ of these abnormal values were only slightly outside of the reference ranges (Table 1, Figure 3).

Consistent with our results, other retrospective and prospective trials examining low-risk elective surgical procedures have demonstrated that the screening tests rarely uncover a disease in asymptomatic otherwise-healthy patients and that the abnormalities found on these tests, if any, very infrequently affect perioperative management. ${ }^{1-3,6}$ In 1997, Narr and colleagues ${ }^{5}$ concluded that patients determined to have no indication for laboratory testing by clinical history and physical examination can safely undergo anesthesia and surgery without increased risk of complications. In 2000, Schein and colleagues ${ }^{14}$ reported their results following a randomized controlled trial in which they compared routine and no preoperative testing in patients undergoing cataract surgery. They demonstrated that in this population of typically older patients with a high prevalence of comorbid conditions and associated laboratory abnormalities, there was no difference in outcomes between the 2 groups. Chung and colleagues ${ }^{15}$ performed a randomized, single-blinded, controlled trial in which they showed no increase in perioperative adverse events after ambulatory surgery between patients with no preoperative testing and patients who underwent indicated preoperative testing, as determined by the Ontario Preoperative Testing Grid. These findings suggest that preoperative testing for low-risk ambulatory surgery can be omitted without adversely affecting outcomes, even in patients with comorbid conditions.

The cost of preoperative testing in the United States is estimated at $\$ 18$ billion annually. ${ }^{16}$ Given increasing and appropriate focus on value-based healthcare, many clinicians and policymakers are focusing on methods to improve efficiency and lower costs without adversely affecting patient outcomes. In our cohort, none of our cancellations were attributed to abnormal results of their PT, PTT, international normalized ratio, Chem7, ECG, and CXR. The total estimated charge for all of these completed tests during the study period was $\$ 2,056,054$. At our institution, all of our patients with congenital heart disease undergo ECG and CXR as part of their initial cardiology evaluation, which poses the question of the need to repeat these studies at the time of surgical intervention if there has been no significant interval change in the patient's clinical status since their last ECG and CXR. This would translate into a decreased cost burden to patients and institutions.

Within our cohort, RLTs revealed significantly elevated potassium and glucose levels in a few patients. Repeat tests were performed because of suspicion of hemolysis or contaminated sample, and in those repeat tests values fell within normal ranges, or were less significantly elevated. Interestingly, those values that did not undergo repeat testing, or those that remained elevated after repeat testing, were not relevant clinically to change the decision to go to the operating room. Our data suggest that even when an abnormal test is significantly outside of the reference range, they do not take an important role in the course of action, and only increase costs and unnecessary testing. Other factors, such as the patient's overall clinical picture and history, should be taken into account to avoid a greater economical and psychological burden to the patient.

\section{Limitations}

This study has multiple limitations, mainly related to its retrospective nature, and the fact that is the result of a single institutional experience; as such, results may vary in different institutions. Furthermore, only hospital charges were available to be used rather than hospital costs. The primary aim of this study was to evaluate the role of RLT has in elective pediatric cardiothoracic surgery in terms of cancellations without taking into account perioperative management, complications, or outcomes.

\section{CONCLUSIONS}

RLT does not appear to significantly impact decisionmaking in elective pediatric cardiothoracic surgery. The decision to order a specific screening test should be clinically driven by physicians and other health providers with the aid of a thorough clinical history and physical examination to avoid unnecessary surgical cancellations and patient discomfort. Selective preoperative laboratory testing may have a positive impact on healthcare costs without affecting outcomes. A prospective trial is warranted to further elucidate the impact RLT has on perioperative management and outcomes in this complex patient population.

\section{Conflict of Interest Statement}

Authors have nothing to disclose with regard to commercial support.

\section{References}

1. Kaplan EB, Sheiner LB, Boeckmann AJ, Roizen MF, Beal SL, Cohen SN, et al The usefulness of preoperative laboratory screening. JAMA. 2002;253:3576-81. 2. Smetana GW, Macpherson DS. The case against routine preoperative laboratory testing. Med Clin North Am. 2003;87:7-40. 
3. Pasternak LR. Pre-operative laboratory testing: general issues and considerations. Anesthesiol Clin North America. 2004;22:13-25.

4. American Society of Anesthesiologists Task Force on Preanesthesia Evaluation. Practice advisory for preanesthesia evaluation: a report by the American Society of Anesthesiologists Task Force on Preanesthesia Evaluation. Anesthesiology. 2002;96:485-96.

5. Narr BJ, Warner ME, Schroeder DR, Warner MA. Outcomes of patients with no laboratory assessment before anesthesia and a surgical procedure. Mayo Clin Proc. 1997;72:505-9.

6. Meneghini L, Zadra N, Zanette G, Baiocchi M, Giusti F. The usefulness of routine preoperative laboratory tests for one-day surgery in healthy children. Paediatr Anaesth. 1998;8:11-5.

7. Turnbull JM, Buck C. The value of preoperative screening investigations in otherwise healthy individuals. Arch Intern Med. 1987;147:1101-5.

8. Brown SR, Brown J. Why do physicians order unnecessary preoperative tests? A qualitative study. Fam Med. 2011;43:338-43.

9. Benarroch-Gampel J, Sheffield KM, Duncan CB, Brown KM, Han Y, Townsend CM Jr, et al. Preoperative laboratory testing in patients undergoing elective, low-risk ambulatory surgery. Ann Surg. 2012;256:518-28.

10. O'Connor ME, Drasner K. Preoperative laboratory testing of children undergoing elective surgery. Anesth Analg. 1990;70:176-80.

11. White B. Diagnosis and treatment of urinary tract infections in children. Am Family Phys. 2011;83:409-15.

12. Gregory GA, Andropoulos DB. Gregory's Pediatric Anesthesia. Chichester, West Sussex: Wiley-Blackwell; 2012.

13. McComb BL, Chung JH, Crabtree TD, Heitkamp DE, Iannettoni MD, Jokerst C, et al. Expert Panel on Thoracic Imaging. ACR Appropriateness Criteria Routine Chest Radiography. Reston, VA: American College of Radiology (ACR); 2015.

14. Schein OD, Katz J, Bass EB, Tielsch JM, Lubowski LH, Feldman MA, et al. The value of routine preoperative medical testing before cataract surgery. Study of Medical Testing for Cataract Surgery. N Engl J Med. 2000;342:168-75.

15. Chung F, Yuan H, Yin L, Vairavanathan S, Wong DT. Elimination of preoperative testing in ambulatory surgery. Anesth Analg. 2009;108:467-75.

16. Kumar A, Srivastava U. Role of routine laboratory investigations in preoperative evaluation. J Aneaesthesiol Clin Pharmacol. 2011;27:174-9.

Key Words: preoperative screening, routine blood testing, routine coagulation screen, preoperative laboratory correspondence

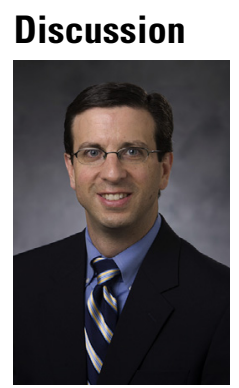

Dr A. Lodge (Durham, NC). Congratulations, Dr Nieto, on a clearly presented and compelling talk. You and your colleagues from Texas Children's Hospital have shown us convincing evidence that routine preoperative laboratory testing in elective pediatric heart surgery patients is costly and unnecessary.

A group at our own institution has investigated a similar question in a slightly different cohort of 529 pediatric patients evaluated in the emergency department, 301 of whom had preoperative laboratory testing and subsequently underwent surgery within 48 hours.

In this study, only $29 \%$ of the patients had abnormal laboratory values likely defined differently than in your study. There were no anesthesia-related complications or delays or cancellations of surgery in any of the patients related to laboratory values, and only 3 patients with abnormal laboratory values required blood products. Each of these 3 had an underlying condition that may have predicted the need for transfusion and justified the laboratory studies.

So even in a presumably sicker group of pediatric patients undergoing nonelective, although noncardiac, surgery, it seems to be safe to avoid routine preoperative laboratory testing.

The results of this QI study have led to a change in practice in all of the pediatric surgical subspecialties at our hospital that stopped short of policy but has led to a reduction in routine preoperative laboratory evaluation.

In our own pediatric cardiac surgery practice, our traditional routine was to order a basic metabolic or chemistry panel, a CBC, an ECG, and a CXR. Six months ago, we changed our practice and have eliminated routine blood work other than a type and screen and have avoided the CXR and ECG if the patient had already had one. We have not studied the result of this change, but it does not seem to have affected our results adversely. As a result of your study, I think we'll look at our practice even more carefully.

I have 2 questions. The first is related to how ingrained this practice is. Have you seen or do you anticipate difficulty with compliance with a policy to eliminate routine testing locally or nationally?

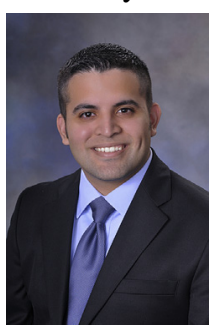

Dr Nieto. I'm sorry, I couldn't hear the beginning of that question.

Dr Lodge. It relates to how ingrained the practice of routine preoperative laboratory testing is. Do you anticipate any problem with compliance with instituting a policy or a practice change to eliminate the testing?

Dr Nieto. I do believe there will be a little bit of pushback with any sort of policy change. There are always different reasons to continue ordering these routine screening tests. One of these being that physicians may have different views on what is a clinical indication.

I think some of these definitions just need to be better defined so they're not too vague and too open to interpretation.

Dr Lodge. The second question relates to specific populations. We've begun to routinely test neonates and infants for fibrinogen and antithrombin 3 levels to try and enhance anticoagulation intraoperatively and postoperative hemostasis. Do you have or think there should be any populations that should have specific testing?

Dr Chen. Populations that you would suggest specific testing for.

Dr Nieto. I'm sorry, populations?

Dr Lodge. So we routinely get a chemistry, a CBC, these other things, but you've stated that that can probably be eliminated safely. There are specific populations of interest, for example, in our practice neonates and infants that we've not necessarily done the routine testing but have looked more specifically at antithrombin 3 levels and fibrinogen levels preoperatively. 
Is there anything like that that you would incorporate into your recommendations for laboratory screening?

Dr Nieto. Well, yes. In this study, the routine tests were for screening purposes where there was no clinical indication to order them. In some certain pediatric populations, there is a clinical indication such as in patients with Down's syndrome. We'll check their thyroid levels as well as order other tests with certain syndromes. But at that point, the screening tests are more clinically indicated and not just ordered routinely.

Dr Lodge. Have you translated these results into a practice or a policy change at Texas Children's?

Dr Nieto. Not yet since this study was a retrospective review. We're looking at collaborating with our laboratory medicine colleagues and moving forward with a prospective observational study to look a little bit more in-depth at the change in perioperative management due to abnormal laboratory values and a little bit more at our outcomes looking at our complications.

Dr Lodge. Thank you.

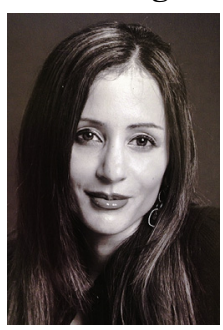

Dr T. Karamlou (San Francisco, Calif). Nice study. I just wondered why you chose to use charges rather than hospital costs, which are more reflective of what the cost of care actually is.

Dr Nieto. I contacted our financial department, and basically they only gave me the charges to work with.

Dr Karamlou. It might be interesting to find out if you can get that data because I think it would be important.

Dr Nieto. I think moving forward with our prospective study, I think we'll be looking at our costs. Thank you.

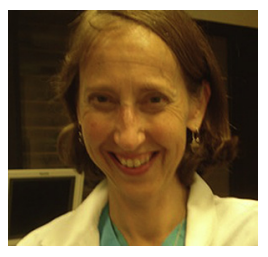

Dr K. Fenton (Memphis, Tenn). I have 2 really quick questions. First, we've had experience in developing countries where, particularly with children with a high white count, sometimes we feel pressured to go ahead and operate on the kid anyway because the child is not going to have another opportunity anytime soon. We have noticed some bad outcomes in those children, in the absence of any other clinical findings. Obviously we decided to operate on the kid because they didn't look sick.

So we've actually gone the opposite direction and made the decision to really reassess and try to not operate on children with an abnormal lab.

So I am kind of wondering if you have any other follow-up on the outcome of patients with abnormal laboratory values that you did operate on.

Dr Nieto. In this study, we were mainly focused on the early decision making in scheduling these patients, and it's going to be in our prospective study, where we will be able to look more in detail at these postoperative outcomes of these patients that had abnormal laboratory values.

Dr Fenton. Okay, thanks. The other thing was a related question: Were there things that you went and fixed? If the patient had an abnormal potassium, for example, maybe you didn't cancel the surgery, but you gave a potassium bolus or transfused the patient or did something to make them a better candidate for surgery. Thank you very much.

Dr Nieto. So we didn't look at every single patient, but for that, we calculated our interquartile ranges for all abnormal laboratory values, and we looked at the outliers for each individual laboratory. In these patients that were significantly elevated, in which you would think something may have been done, nothing was really done outside of maybe repeating the labs. Like the potassium, for example, they were all hemolyzed. Blood glucose levels that were elevated were just repeated because the patient may have had a candy or something else to eat or drink.

One thing that I can recall, for our UAs, there were a handful of abnormal UAs that were suggestive of UTIs, and these patients did receive antibiotics and proceeded on with surgery. Some were culture-positive and some were not. 